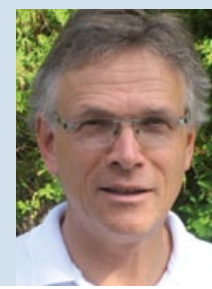

Dr. med.

Ulrich

Mutschler,

Hildesheim

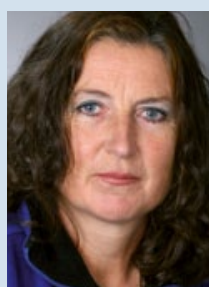

Dr. med.

Kirsten

Stollhoff,

Hamburg

\section{Hausgeburt verdreifacht neonatale Sterblichkeit}

Immer wieder wird diskutiert, Geburten außerhalb des Krankenhauses stattfinden zu lassen - mit dem Hinweis, dass bei Müttern mit niedrigem Risiko die Entbindungen zuhause eine sichere Angelegenheit sei. Eine aktuelle Studie zeigt jedoch alarmierende Ergebnisse oseph Wax vom Maine Medical Center aus Portland, USA, und sein Team haben eine umfangreiche Metaanalyse vorgelegt: Sie verglichen darin die Komplikationsraten bei geplanten Haus- und Klinikentbindungen. Die Autoren werteten zwölf Studien mit insgesamt etwa 500.000 Geburten aus sechs verschiedenen Ländern (USA, Kanada, Australien, Schweden Niederlande und Schweiz) aus. Die Häufigkeiten der Hausgeburten in diesen Ländern sind sehr unterschiedlich: In den Niederlanden beträgt der Anteil $30 \%$, in den USA dagegen nur etwa $1 \%$ der Geburten im Jahr.

Die Hausgeburt hatte für die Mutter zwar einige Vorteile: weniger Dammrisse, Blutungen und Infektionen. Der entscheidende Nachteil war jedoch eine Verdreifachung der neonatalen Mortalität $(0,15$ vs. $0,04 \%$, Odds Ratio: 2,87). Die Autoren kommen zu dem Schluss, dass bei der Hausgeburt die niedrigeren Raten an Komplikationen bei der Mutter überschätzt und die Risiken für das Kind unterschätzt werden.

Kommentar: Die Studie wird in der renommierten Zeitschrift Lancet kommentiert (Lancet 2010; 376: 303). Es heißt dort: „Frauen haben das Recht zu wählen, wie und wo sie gebären; sie haben aber nicht das Recht, ihr Baby einem Risiko auszusetzen. "In bestimmten Situationen mag die Hausgeburt eine mögliche Option für Mütter mit unkomplizierter Schwangerschaft sein - vorausgesetzt, sie sind über die Risiken aufgeklärt und die Hebamme verfügt neben ihren Fähigkeiten in der Geburtshilfe auch über Geschick in der Wiederbelebung von Neugeborenen. Zusätzlich ist es erforderlich, dass die Gebärende im Bedarfsfall einen raschen Zugriff auf eine klinische Geburtshilfe hat.

Dr. Hartmut Koch

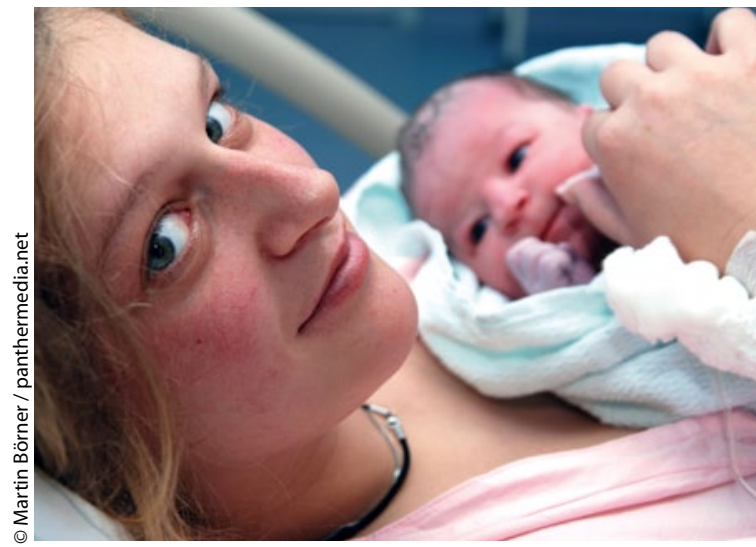

Im Krankenhaus zu entbinden, ist für das Kind noch immer am sichersten.

Wax JR et al. Maternal and newborn outcomes in planned homebirth vs. planned hospital births: a metaanalysis. Am J Obstet Gynecol 2010; 203: 243.e1-8

\section{Weniger Asthma per Gesetz}

Die Zahl der Krankenhauseinweisungen von asthmakranken Kindern sinkt pro Jahr um knapp ein Fünftel, seit es Gesetze zu öffentlichen Rauchverboten gibt. Dies hat eine Studie aus Schottland ergeben, wo das Gesetz schon im März 2006 in Kraft getreten ist. Daniel Mackay und Kollegen von der University of Glasgow hatten alle 21.415 Klinikeinweisungen in Schottland von 2000 bis 2009 analysiert, die Kinder unter 15 Jahren mit der Diagnose „Asthma“ betroffen hatten. Vor der gesetzlichen Einführung des öffentlichen Rauchverbots war die Zahl entsprechender Einweisungen jährlich im Mittel um $5,2 \%$ gestiegen. Danach sank die Rate um 18,2\% pro Jahr.

Kinder im Vorschulalter sind dem Rauch vor allem im Elternhaus ausgesetzt. Schulkinder hingegen sind häufiger in der Öffentlichkeit unterwegs und fangen womöglich selbst an zu rauchen. Auch hier wird durch das Gesetz offenbar ein Riegel vorgeschoben: Die Rate von 13-jährigen Jungen, die selbst rauchen, fiel von 5\% im Jahr 2004 auf 3\% 2007. Bei den gleichaltrigen Mädchen war ein Rückgang von 7 auf $4 \%$ festzustellen. 